Teaching experience in urban modeling

\section{| RESUMEN}

Se presenta una experiencia docente en forma de curso semestral llevada a cabo con alumnos de $4^{\text {to }}$ año de la carrera de Arquitectura en la Universidad de Chile. El curso se enfoca en metodologías de modelamiento urbano que permiten abordar fenómenos desde un enfoque de abajo hacia arriba (desde el modelamiento de las decisiones de los individuos, para luego observar cómo emergen patrones a mayor escala a partir de la simulación), lo que permite abordar de forma explícita la complejidad de la ciudad. En este artículo se aborda una discusión sobre la necesidad de exponer a estos modelos a los alumnos, luego se detallan los modelos utilizados (Economía Regional, Segregación, Visibilidad, Evaluación Multicriterio, entre otros), para finalizar con algunos criterios de evaluación y perspectivas a futuro. Se concluye haciendo énfasis en la importancia de incorporar nuevas herramientas de análisis cuantitativo para respaldar decisiones, pero teniendo en cuenta que no son herramientas predictivas ni reemplazan la opinión experta.

\section{| ABSTRACT |}

This article presents a one semester teaching experience conducted with $4^{\text {th }}$ year architecture students at Universidad de Chile. The course focuses on urban modeling methodologies that tackle phenomena from a bottom-up approach (modeling the decisions of individuals, in order to observe how patterns emerge on a larger scale, using simulation), which allows to explicitly address the complexity of the city. A discussion is presented on the need to expose students to these models, then the models used are detailed (Regional Economy, Segregation, Visibility, Multiple Criteria Decision Analysis, etc.), followed by with review criteria and perspectives. It concludes by emphasizing the importance of incorporating new tools of quantitative analysis to support decisions, but considering they are not predictive tools or replace expert opinion.

\title{
Experiencia docente en modelamiento urbano
}

\author{
Un buen viajero \\ no tiene planes precisos \\ ni la intención de llegar. \\ Li Er / Lao Tsé
}

\section{LA ENSEÑANZA DEL URBANISMO: ESCALANDO POR CAMINOS FRECUENTADOS HACIA LA EXPERIMENTACIÓN CREATIVA}

No hay duda que en el encuadre de la hegemonía neoliberal afrontamos un conjunto de condiciones que tienden a menoscabar o cuando menos prescindir de la eficacia y rigor de la práctica urbanística, y que esta situación viene determinando, además de cierta falta de convicción respecto la calidad del ejercicio profesional, un evidente desconcierto ante la necesidad de formalizar los objetivos y métodos de enseñanza de la disciplina.

Primero, se han acrecentado (como nunca antes) las oportunidades de acceso a la información emitida por fuentes cada vez más diversificadas, tanto de los especialistas como de los ciudadanos en general, potenciado su acercamiento a un sinnúmero de referencias y ambientes que no obstante son virtuales poseen una riqueza y facilidad de interpretación que se multiplica permanentemente.

Del mismo modo se ha venido ampliando la capacidad de incorporar datos, traducirlos en variables, clasificarlos y correlacionarlos siguiendo pautas cada vez más apropiadas a la búsqueda de las significaciones que aporta la divulgación de programas estadísticos más asequibles y rutinarios.

Por otra parte, en el lenguaje corriente y especialmente gracias a las argumentaciones de contrastación que circulan por el ámbito político, se ha generalizado el concepto de sistema y sus dinámicas de interrelación, facilitando el manejo de los análisis de causalidad, y la difusión de

Arquitecto, Universidad de Chile (2007), Magíster en Geografía y Geomática, Universidad Católica (2013). Profesor Ayudante Departamento de Urbanismo, Universidad de Chile.

** Arquitecto, Director del Departamento de Urbanismo de la Facultad de Arquitectura y Urbanismo de la Universidad de Chile. Ha desarrollado su labor académica como investigador, docente de pre y posgrado, y proyectista en Urbanismo. Sus aportes más recientes se articulan en torno a la evolución de los paradigmas que guían el proceso chileno de urbanización y, en ese encuadre, alrededor de la dinámica de la interfaz urbano rural, y sobre la lógica de perfilamiento y expresión secuencial de la ciudad neoliberal. 
las técnicas de marco lógico en el juego de las decisiones, configuración de escenarios y seguimiento ahora reiterado de los proyectos de intervención.

La misma vertiente ha impulsado un giro en el enfoque de las problematizaciones, alentando cambios que van desde la búsqueda de certezas y viabilidades de tono positivista, hacia la consideración de la fuerza (cuotas de poder) y capacidad de negociación de los agentes conflictuados, de tono probabilístico.

El compromiso de la sociedad con el proceso de urbanización ha sido animado por la edición de «observatorios» oficiales e independientes, a través de los cuales se difunden materiales procedentes del trabajo analítico y proyectual. Y hay también una cuota de estimulación, limitada todavía, que deriva del empleo de sistemas de información en algunos municipios pioneros, así como técnicas de modelamiento predictivas y didácticas para simular intervenciones urbanísticas montadas sobre instrumentos que semejan la complejidad y dinámica de la realidad (Ruiz-Tagle, Gurovich \& (ox).

Ante las restricciones que impone un paradigma imperante ligado a metodologías carentes de rigor y contenido, el urbanista debe insertar metodologías validables que permitan mediar entre las instancias de decisión y las comunidades afectadas. Teniendo esto en cuenta, el curso descrito se interpreta desde algunas propuestas relativas a dos ámbitos: la realidad y la intervención del medio.

Respecto a la primera, resalta un artículo de Enrique Florescano que muestra como el valor relativo de la realidad (presente) se modifica debido a determinada visión del pasado (reforzada por cierto dominio políitico) (Florescano, 1980, 2005). En este sentido es importante plantear que la sociedad se está constantemente inventando a sí misma, afectando su orden y entendimiento, en una dicotomía entre lógicas heredadas y el imaginario social en el conocimiento de la realidad (Castoriadis, 1975).
Por último, la conciencia de intervención del medio se reinstala en nuestro tiempo, ya francamente en la vertiente lefebvreriana y multidimensional en las obras de David Harvey (1998) sobre la producción espacial en la posmodernidad; los artículos de Ana María Rigotti (1996), quien reivindica la lógica científica de los expedientes urbanos; y las publicaciones de Alicia Lindón (2007), a través de las cuales despliega nuevas posibilidades de estudio de los elementos de lo urbano.

Todo esto nos ha venido estimulando en la innovación de la enseñanza del Urbanismo en la Facultad de Arquitectura y Urbanismo de la Universidad de Chile, teniendo en cuenta que hoy en día es necesario y más aún es posible transitar hacia modelos que permitan explicitar el discurso de la complejidad de las ciudades actuales.

\section{APROXIMACIÓN A LA COMPLEJIDAD DE LAS CIUDADES}

Michael Batty (1995) hace ya casi 20 años daba cuenta del cambio de paradigma que se ha venido produciendo en el estudio de las ciudades, desde una visión de arriba hacia abajo (top-down) hacia una de abajo hacia arriba (bottom-up). Esto se refiere a modelar las ciudades desde las interacciones más sencillas de quienes toman decisiones en el territorio, para luego observar los fenómenos a escala macro. De abajo hacia arriba implica hacer la relación entre lo local y los patrones que se pueden observar a gran escala. Batty planteaba que los modelos gravitacionales (interacciones entre centros poblados) estaban dando paso a modelos desagregados que permiten explicar este mismo tipo de fenómenos regionales, pero esta vez desde modelos evolutivos que reproducen la autoorganización de agentes en centros a diferentes escalas y jerarquías.

Esto de la misma forma que en diferentes ramas de la ciencia que han tomado el camino del modelamiento a partir de las interacciones básicas. El paradigma de los Sistemas
Emergentes ha ido expandiéndose como una forma efectiva de representar la realidad (Johnson, 2001). Este paradigma, como una herramienta de análisis, se basa en observar los fenómenos como un producto de múltiples agentes interactuando en base a reglas sencillas pero produciendo fenómenos complejos, los cuales no son guiados por un control exterior, sino solo por la sincronicidad de sus elementos más básicos (Johnson, 2001). De esta forma es posible entender procesos urbanos de aglomeración, complementareidad (clusters), competencia y especialización, entre muchos otros (Krugman, 1991).

A pesar de este cambio de paradigma, Batty (1995) advertía que la mayoría de la planificación no se realizaba considerando una visión desde abajo hacia arriba. Actualmente, sobre todo en nuestro país, es posible observar que el diseño de instrumentos de planificación no considera ni el diagnóstico ni el ordenamiento territorial desde una perspectiva sistémica y emergente, sino más bien por partes agregadas que interactúan de forma lineal y predecible a partir del juicio experto y no a partir del modelamiento.

Cambiar el paradigma se hace necesario toda vez que la estructura de la ciudad chilena actual se ha hecho cada vez más descentralizada, aumentando el poder de las decisiones individuales en la conformación de la estructura metropolitana, llevando la ciudad a organizaciones de mayor complejidad (Borsdorf, 2003; De Mattos, 2001).

\section{LA CIUDAD COMO UN FENÓMENO COMPLEJO PERO MODELABLE}

Los modelos urbanos aspiran a representar la complejidad de una ciudad mediante una reducción operativa de la realidad. Es una reducción ya que es necesario sintetizar la información agregando elementos en jerarquías mayores. Esta síntesis es necesaria para su computabilidad, si no no tendría sentido y estaríamos frente al fenómeno mismo. Es operativo ya que permite su manejo en 
diferentes escenarios para explorar escenarios alternativos y comunicar los resultados. Y se basa en la realidad ya que se alimenta de información observable y medible.

Pero, ¿̇por qué modelar? Esta pregunta se la hace Epstein (2008), aclarando que el modelamiento no tiene por objeto principal la predicción, si no que se justifica también en función del proceso. De hecho plantea que no necesariamente debe predecir. Uno de los principales aportes es transformar en explícitos los modelos implícitos (de estos últimos, por ejemplo, los discursos sobre fenómenos urbanos). Se transforman en explícitos cuando es posible detallar las variables que definen el comportamiento del fenómeno y las relaciones que hay entre estas. Así se puede pasar de un discurso que puede ser redundante, incompleto y contradictorio, a una lógica formalizada que ayuda a descubrir incoherencias y sugerir nuevas interpretaciones.

De esta forma se pueden sugerir relaciones causales entre variables que antes pueden no haber sido intuidas. De hecho una de las principales características de un modelo es entender qué variables explican la variación de otras variables. Esta propiedad es muy relevante para una propuesta de ordenamiento territorial, para la cual es necesario saber dónde intervenir para maximizar los efectos positivos. Por último, un modelo permite comunicar de forma directa y sin ambiguedades, facilitando la discusión y la posibilidad de ser rebatidos.

Si bien un modelo puede ser muy básico y describir relaciones directas entre pocas variables, el paradigma actual apunta hacia la desagregación de información a nivel de agentes y de células espaciales reducidas (a nivel predial) (Waddell, 2009), lo cual nos permite volver hacia el paradigma de los sistemas emergentes, siendo los modelos desagregados una forma de reconstituir los fenómenos desde los elementos básicos. La desagregación se ha transformado en la corriente más seguida en modelamiento. Esto es coherente con el creciente individualismo, y la diversificación en estilos de vida, patrones de localización y movilidad. Esto se complementa con una mayor disponibilidad de información (Wegener, 2011).

Ante este escenario es importante dar cuenta que las metodologías de diagnóstico urbano generalmente no consideran modelamiento explícito de los fenómenos. Tampoco se considera en la formación de los arquitectos que van a desarrollar diagnósticos. Por lo general se aborda esta disciplina desde una perspectiva agregada, con un enfoque de arriba hacia abajo, de la misma forma que se abordan los encargos a menor escala (Casakin \& Portugali, 2006). En el escenario chileno, es importante aprovechar la tradición nacional en modelamiento urbano, que han aportado modelos que han liderado el estado del arte a nivel mundial, como MEPLAN (Marcial Echeñique), TRANUS (Tomás de la Barra) y MUSSA (Francisco Martínez).

\section{LA INTEGRACIÓN DEL MODELAMIENTO A LA ENSEÑANZA DEL URBANISMO}

Considerando este cambio de paradigma en curso, surge la inquietud de su integración a la práctica del diagnóstico y ordenamiento territorial, en un ámbito en el que se puede tener mayor experimentación, como es el académico.

La experiencia se basa en la introducción de los alumnos a diferentes metodologías de modelamiento y simulación, con dos áreas principales: Teórica y Práctica (una distinción de todas formas ambigüa, pero que ayuda a enfocar el curso).

Los aspectos teóricos y prácticos se enlazan y no son lineales, sobre todo si consideramos que no se busca desarrollar habilidades específicas, sino más bien exponer a los alumnos a otras formas de diagnóstico e intervención urbana. Este proceso del «exponer a» es el que guía la experiencia, y a partir del uso de diferentes formas de modelamiento se busca generar una discusión sobre las formas tradicionales en la práctica del urbanismo.
La línea práctica del curso se basa en modelos que van desde lo abstracto a lo aplicado.

Esto permite hacer la transición entre el modelamiento desde sus componentes más básicos, hasta la aplicación de modelos en base a información más detallada de fenómenos urbanos.

La línea teórica reconoce que la posibilidad de integrar modelamiento a la práctica del diseño y planificación urbana tiene relación con paradigmas con los que se abordan los fenómenos. En este sentido es necesario incorporar una discusión sobre conceptos relacionados con Sistemas Emergentes, pero también sobre disciplinas aplicadas que se han nutrido de esto, como la economía urbana.

Al plantear un curso de este tipo, surge la problemática de la implementación, teniendo en consideración que un modelo urbano como los presentados anteriormente normalmente tienen una complejidad que requiere formación matemática, además de mucho tiempo para recolectar y formatear la información, además de las destrezas para evaluar escenarios e interpretar resultados. En este sentido, se busca en el curso partir desde modelos abstractos pero que sin embargo sean analogías válidas de la realidad.

\section{ANALOGÍA: AGENTES QUE TOMAN DECISIONES EN FUNCIÓN DE SU ENTORNO}

Los modelos aplicados parten de una situación que es natural a los alumnos de arquitectura: la relación entre individuo y espacio. Para esto se introduce a los conceptos de agente y de matrices espaciales. Mediante los modelos se apunta lentamente a integrar el componente de decisión de los individuos (en función de su espacio) como una forma de entender los fenómenos urbanos. A su vez, se presenta el entorno como un espacio dividido en celdas, cada una de ellas con propiedades que varían según su vecindad y/o según la acción de los agentes. 
1. Aplicaciones curriculares del Modelo de localización de Von Thünen y del modelo de segregación de Schelling.

La plataforma utilizada permite el modelamiento de estas dos instancias interrelacionadas. La plataforma utilizada es NetLogo, desarrollada por Uri Wilensky (1999) de la Universidad de Northwestern (Illinois, EE.UU.), con el propósito de generar un entorno para modelar fenómenos complejos a partir de un espacio de celdas (llamados Autómatas Celulares) y un conjunto de individuos (Sistemas Multi Agente).

Los Autómatas Celulares son una representación discreta del espacio, mediante una grilla en la cual cada casilla o celda tiene un valor que varía en el tiempo según el estado de las celdas vecinas. De esta forma se puede representar, por ejemplo, la evolución del valor de suelo, construcción, o localización, pero más que nada se ha usado en otras disciplinas como biología para representar procesos como la reproducción celular o la propagación de un virus.

Sobre esta grilla, NetLogo permite superponer un Sistema Multi Agente, el cual se basa en la representación espacial de los criterios, decisiones y acciones de individuos en un ambiente con propiedades que determinan el actuar de estos. Debido a su capacidad de reproducir fenómenos técnicos y sociales complejos, los modelos multiagente demuestran ser útiles para el entendimiento de fenómenos como las relaciones internacionales, comercio, parentesco, normas, etc (Axelrod, 1997).

Algunas experiencias actuales de modelamiento con SMA van desde la resiliencia en de granieros respecto al cambio de uso de suelo (Pollhill et al., 2010), modelos de la formación de periferias en Latinoamérica (Barros, 2003), comportamiento de compradores y vendedores en el mercado inmobiliario replicando el modelo de Alonso (Filatova et al., 2009), migraciones intra urbanas considerando factores de identificación cultural (Benenson, 1998).

NetLogo permite mediante un entorno adaptable programar las leyes que rigen los cambios de estado de las celdas, y a su vez el comportamiento de los agentes. También permite adecuar la interfaz para poder diseñar las experiencias de simulación y visualizar los resultados.

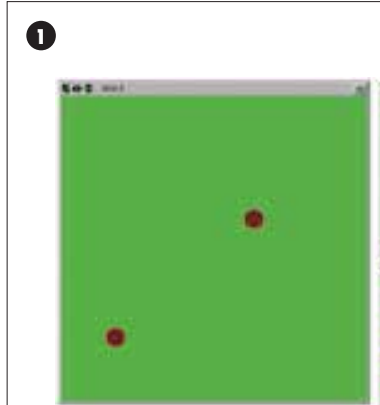

Teoría de localización de Von Thunen
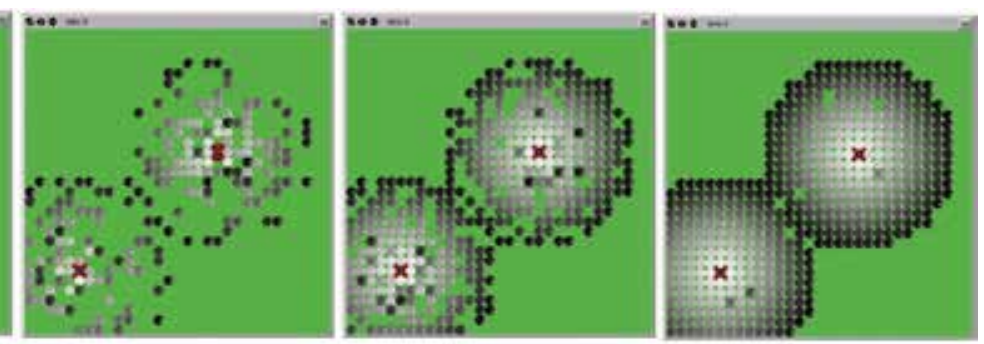

Modelo de segregación de Schelling
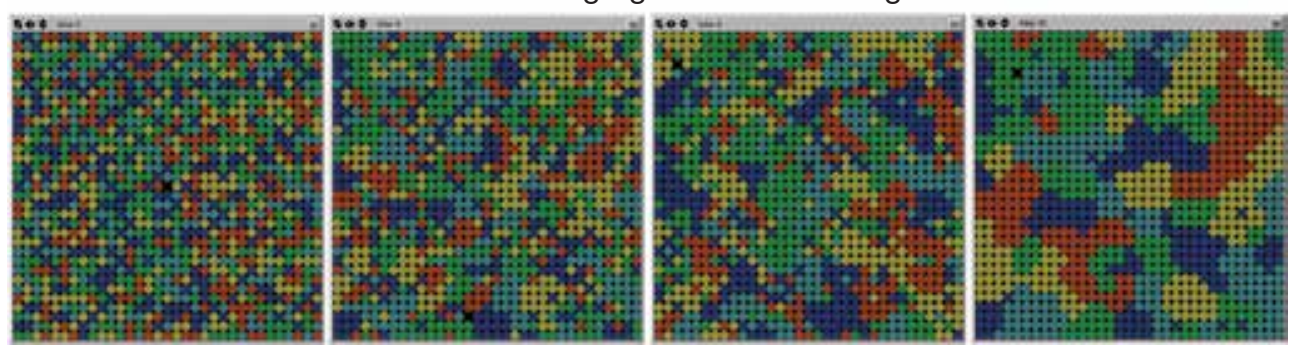

En semestres anteriores los ejercicios del curso se basaban en el uso del juego Sim City como analogía de la ciudad, sin embargo, en el año 2012 se optó por usar NetLogo debido a sus posibilidades de diseñar los algoritmos que dan vida a los procesos de cada modelo, pudiendo variar entre diferentes fenómenos, escalas y niveles de detalle.

\section{DESARROLLO DEL CURSO: DESDE MODELOS ABSTRACTOS A MODELOS APLICADOS}

En este tránsito desde lo abstracto a lo aplicado se inicia la experiencia con uno de los primeros modelos que popularizó a los autómatas celulares a principios de la década de los setenta: El Juego de la Vida de John Conway (Gardner, 1970). Este modelo busca de forma sintética representar en una grilla la evolución de un organismo en un espacio en el cual las células están vivas o muertas dependiendo del estado de su vecindad próxima.

A continuación, una vez entendiendo y observando las interacciones complejas que se pueden dar espacialmente e integrando el factor temporal, se plantean dos modelos que comienzan a integrar variables relacionadas con las decisiones de los agentes. El modelo de Von Thünen (1826) fue uno de los primeros modelos de Geografía Regional, que plantea cómo se distribuye la renta de la tierra en torno a un mercado puntual. Se basa en una fórmula que integra variables de costo de transporte, productividad y utilidades del tipo de producción. El modelo predice que la producción que mayores utilidades entregue, se va a localizar más cercano al mercado. Se modelaron estos supuestos a partir de agentesproductores que buscan la localización más óptima para su tipo de producción, pudiendo observar cómo se verifican las observaciones del autor. Se incluyó posteriormente la posibilidad de modificar algunos parámetros del espacio de producción (tasa de transporte, como si se construyeran caminos y el espacio dejara de ser isótropo, o productividad de la tierra), observando cómo se reorganizan los productores.

Es interesante este tipo de simulación ya que se puede observar cómo a partir de condiciones 
iniciales azarosas (como la localización inicial de los productores), y aplicando la presión del medio (competencia), se establece un orden autoorganizado. Un segundo modelo es el de Duopolios de Hotelling (1929), presentado por su autor para demostrar, en contra de lo que uno podría intuir, cómo a partir de dos productores que compiten por consumidores, se tiende a una localización centralizada de ambos, en vez de distribuirse equitivamente en el espacio. El modelo original de Hotelling era en un espacio lineal (para facilitar el cálculo en el tiempo), pero contando con NetLogo se pudo observar cómo se daba este fenómeno en un espacio 2D, y sin la necesidad de calcular manualmente, con lo cual se puede experimentar en diferentes escenarios de forma fácil y rápida. Este modelo es interesante ya que se extiende no solo a economía espacial, sino también a entender por qué los productos tienden a homogeneizarse y en general lo diferente no es competitivo, como planteaba el autor en su artículo original.

Posteriormente, el modelo de Segregación de Schelling (1979), presentado por Thomas Schelling como una forma de demostrar cómo ciertos patrones en escala macro pueden diferir de los criterios que guian los comportamientos a escala personal. El modelo simula las decisiones de localización de dos grupos (pueden ser etnias, grupos socioeconómicos, etc.), que son relativamente tolerantes en cuanto a la composición de su vecindario (tienen como estándar de buena localización el que el 30\% de sus vecinos sean del mismo grupo, o si no se sienten incómodos y se mudan), sin embargo, al simular en el tiempo, el equilibrio (que todos están conformes con su localización) Ilega solo cuando hay dos zonas totalmente segregadas en el espacio.

Este es un ejemplo de un sistema en el cual «emergen» patrones que no son intuibles a partir de la observación desagregada de los factores (similar al concepto de sinergia), y parecido a lo que sucede en una colonia de hormigas o en el cerebro a partir de una red de neuronas. Este modelo fue construido con la posibilidad de modificar el porcentaje de vecinos similares
2
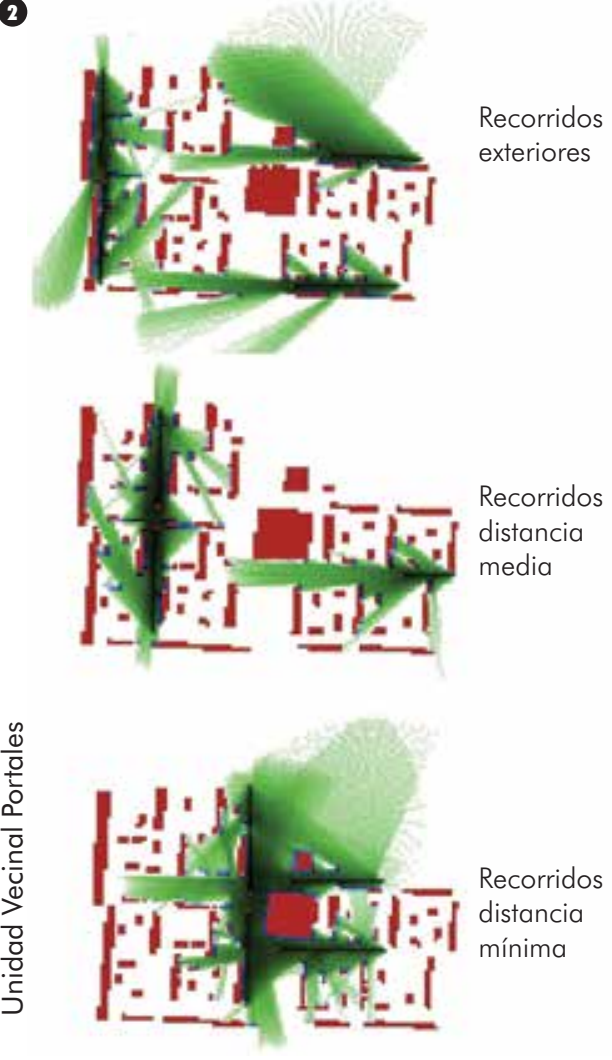

Cristián Aránguiz

necesarios para que una persona esté feliz en su localización, con el objeto de poder observar fácilmente cómo esto influye en la distribución final en la ciudad.

Estos primeros modelos tienen un carácter más abstracto y para los cuales no se consideran casos específicos que puedan ser propuestos por los alumnos. Su función también es interesante como una introducción a modelos clásicos de economía urbana que normalmente se enseñan en pizarrón.

A medida que avanza el semestre se presentaron modelos que permitían una mayor incorporación de información real por parte del alumno, para posteriormente evaluar los resultados.

Se diseñó un modelo de flujos en un terminal de buses, mediante el cual se pueden explorar dinámicas complejas de encadenamientos de

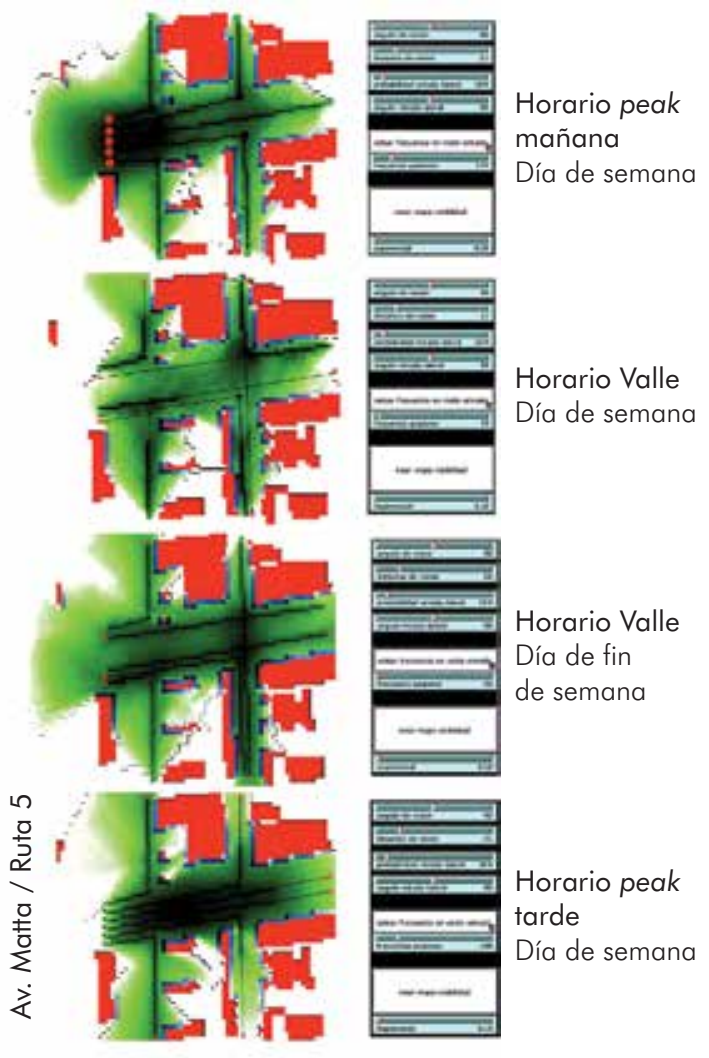

Rafael Parra

procesos y relación no-lineal entre variables. Por ejemplo, si es necesario transportar más pasajeros no basta con aumentar la llegada de buses, porque si esto no se acompaña con aumento de la cantidad de andenes o del área de tránsito, puede implicar congestión y producir el efecto contrario (menor cantidad de pasajeros saliendo). La observación de variables de salida (cantidad de pasajeros transportados) permite evaluar la efectividad de diferentes diseños

Con una mayor posibilidad de integrar un entorno real a partir de un mapa Nolli, se implementó un modelo de visibilidad. Jane Jacobs releva la importancia del control que ejercen los ojos de la calle en la seguridad de los espacios públicos, marcando la diferencia entre un espacio propicio para estar y uno que se degrade y sea subutilizado. Hay algunos modelos que permiten evaluar espacios desde 
3. Evaluación Multicriterio de la localización de comercio vecinal en el entorno del edificio Costanera Center (C. Cerda, I. Novoa, primavera 2012).

la perspectiva de las líneas de visión, aperturas y proporciones de los espacios (por ejemplo la metodología de Space Syntax), sin embargo, el fenómeno es complejo ya que los conos de visión varían según la posición de los peatones y las direcciones de su movimiento. Se construyó un modelo que permite simular el recorrido de los peatones en un mapa Nolli del área de estudio, asignando frecuencias para jerarquizar vías principales y secundarias, y que también permite determinar la amplitud de los conos de visión y frecuencia de miradas laterales, entre otros parámetros. La información que entrega el modelo permite observar los espacios más o menos expuestos a la visión de los peatones, así como también las fachadas que tienen más presencia. Este último punto es otra característica que entrega información relevante, ya que permite estudiar superficies comerciales o de publicidad.

Los últimos dos modelos, si bien permiten la incorporación de información de un contexto urbano específico, tienen la función de mostrar dinámicas no lineales más que servir como instrumentos operativos de evaluación, dada la complejidad de las variables consideradas (movimiento de peatones).

A continuación, finalizando el semestre, se aplican dos modelos que tienen mayor validez, y que son ampliamente aplicados. Su carácter más preciso y validado sacrifica la posibilidad de observar comportamientos emergentes como los observados en los primeros modelos.

El primero de estos modelos busca evaluar la Accesibilidad en un entorno urbano. Se construyó un modelo que permite a los alumnos la evaluación de redes de recorridos, considerando fuentes o centros, y espacios a los cuales era necesario acceder. Todo esto ingresando la red de recorridos como una imagen raster en la que cada recorrido era cargado con una fricción o fuerza (por ejemplo, una calle puede tener un valor base de 1 , pero un parque puede tener fricción de 1,5 , y a su vez una masa construida tiene una fricción cercana a infinito). Esto permitió, a base de un algoritmo de spill o derrame, evaluar los recorridos
3

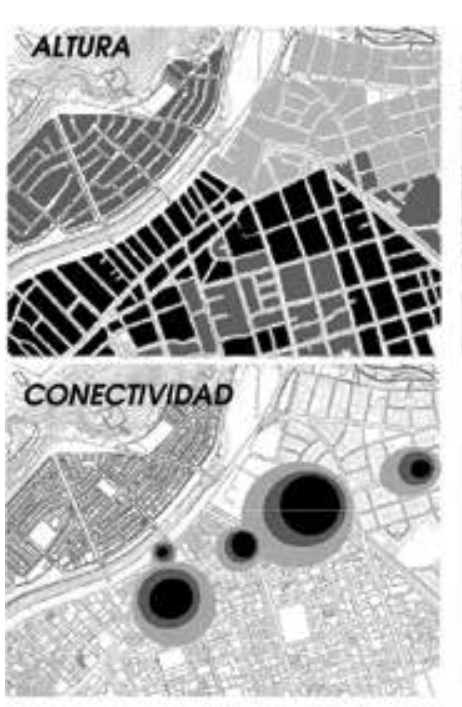

Criterios:
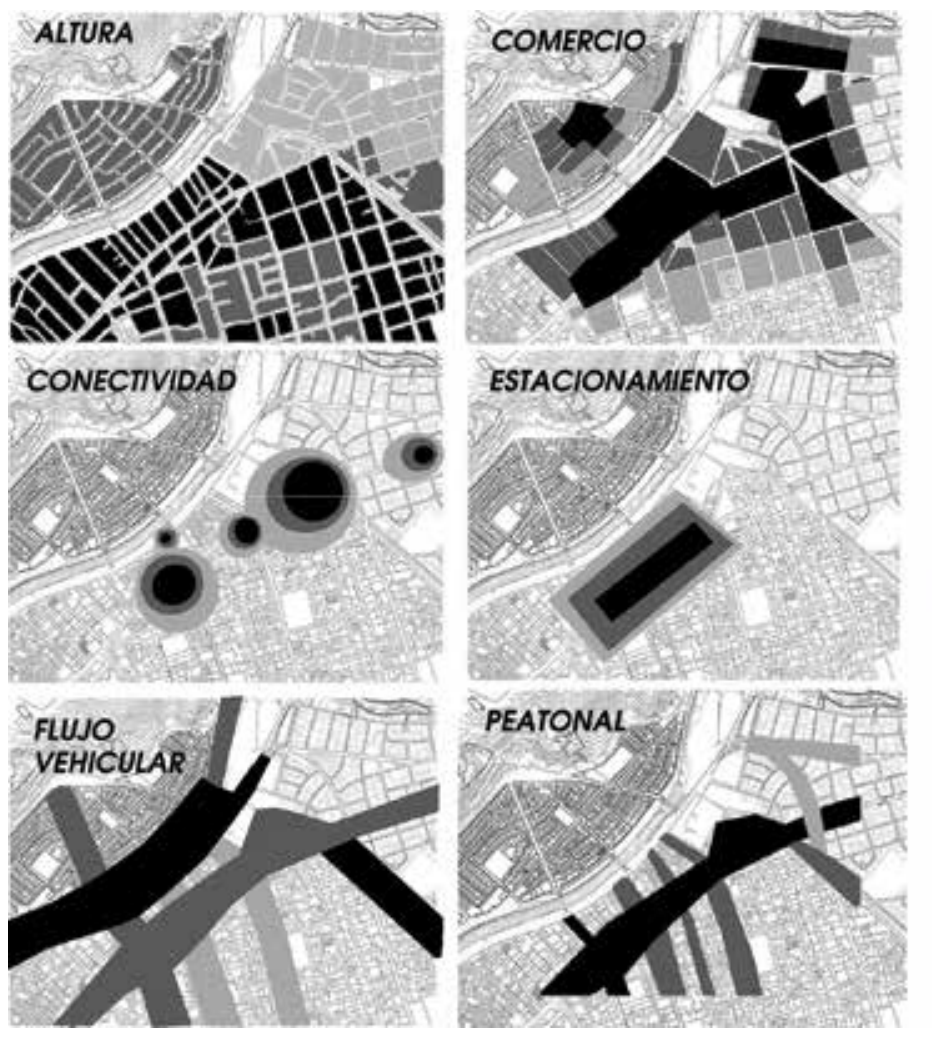

Resultados por escenarios:

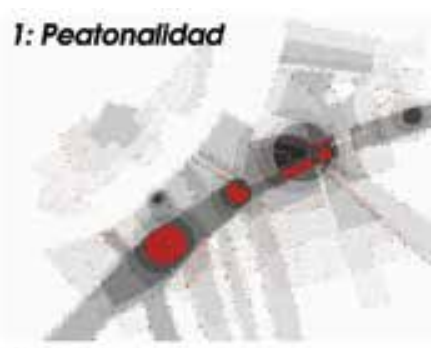

2: Vehicular
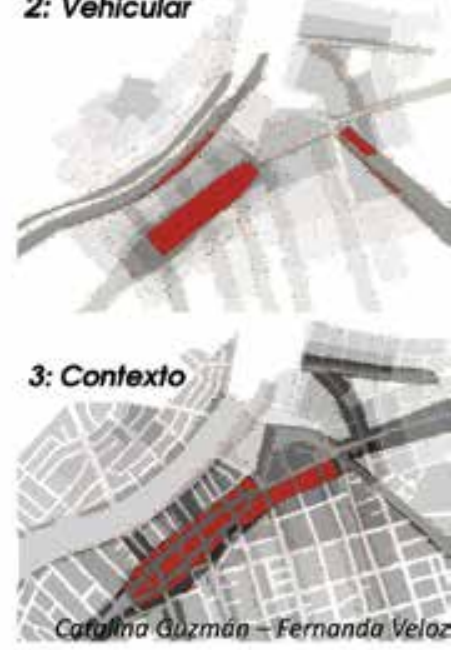

óptimos para determinar accesibilidad. Este modelo permite evaluar los beneficios de la apertura de una calle, o evaluar localización para un equipamiento $u$ otro tipo de usos.

El último modelo es más bien una herramienta, que busca representar los criterios de decisión en la localización, conocido como Evaluación Multicriterio. Esta metodología ha sido desarrollada por diferentes autores (Keeney \& Raiffa, 1976; Saaty, 1980; Roy \& Vanderpooten, 1996; Malczewski \& Ogryczak, 1995), como una ayuda a la toma de decisiones en base a criterios y a ponderaciones de importancia para cada uno de estos criterios. En este caso específico se aplica para la decisión de localización de un equipamiento a elección del alumno, en la cual los criterios son espacializados y se representan como aptitud de recibir ese equipamiento. Este modelo permite ingresar la información entregada por los modelos de accesibilidad y de visibilidad, como factores para evaluar.

\section{EVALUACIÓN Y OBJETIVOS DE APRENDIZAJE}

Como se presentó en un principio, el objetivo es exponer a los alumnos a formas de modelamiento, como una alternativa al diagnóstico tradicional en urbanismo. Durante el curso no se ha desarrollado una forma sistemática de registrar y medir los conocimientos adquiridos por los alumnos. Las maneras de poder evaluar la incorporación de los contenidos es compleja, ya que lo que el alumno ve en este curso se sale de la línea acostumbrada en los cursos anteriores de urbanismo. Por lo tanto, se espera que al final del semestre el alumno tenga una mapa de los contenidos y de lo que implica modelar (que 
no le sea algo ajeno), más que desarrollar una capacidad aplicada.

Sin embargo, algunos de los objetivos de aprendizaje que guían la pertinencia de los modelos expuestos se refieren a que el alumno pueda:

- Formular un discurso coherente sobre la importancia del modelamiento y simulación, sus limitaciones, campos de aplicación, recursos necesarios, y resultados esperables.

- Manejar de forma básica conceptos de economía urbana: modelos clásicos de geografía regional, mercado inmobiliario, segregación urbana, transporte.

- Desglosar un fenómeno urbano en variables medibles y establecer hipótesis de relaciones causales entre las variables.

- Manejar y saber utilizar conceptos de modelamiento y diseño experimental: Variables, hipótesis, correlación, causalidad, escenarios.

- Manejar conceptualmente los pasos básicos de metodologías específicas de modelamiento y simulación: modelos de accesibilidad, modelos de localización, modelos en base a autómatas celulares, modelos en base a multiagentes.

La evaluación del aprendizaje se basa más que en la efectividad de los resultados de los modelos, en la capacidad de los alumnos de desarrollar documentos coherentes con reflexiones y entendimiento de las posibilidades y limitaciones del modelamiento.

\section{DESARROLLO ACTUAL DEL CURSO}

En el semestre en curso (otoño 2013), se ha integrado un nuevo profesor, Ricardo Hurtubia, lo que ha permitido reforzar de manera crítica los contenidos del curso. Si bien las bases en cuanto a sistemas emergentes se mantienen, se ha buscado enfocar el trabajo semestral en un caso específico de estudio a escala metropolitana, con capas georreferenciadas de atributos (escuelas, estaciones de metro, etc.), e información censal de hogares, lo cual permite estimar modelos que explican la localización de los hogares en función de los atributos. Este enfoque se basa en un modelo de elección discreta (Ben Akiva \& Lerman, 1985 Anas, 1982), que si bien se puede aplicar para cualquier tipo de elección, para este caso permite dar luces sobre las motivaciones de las personas en la búsqueda de la localización de sus hogares, lo cual es de vital importancia para entender el futuro de nuestros territorios.

La línea actual permite exponer a los alumnos a fenómenos en base a información real y que tengan un acercamiento más directo a metodologías ampliamente validadas y utilizadas actualmente en modelamiento de los usos de suelo y transporte.

\section{CONCLUSIONES Y COMENTARIOS}

Los modelos permiten el entendimiento de la ciudad como un sistema, basado en fenómenos complejos, pero medibles y modelables con la incorporación de la simulación (el factor temporal), y la aplicación de conceptos que nos entregan las teorías en Sistemas Emergentes (y otras anteriores también revisadas como Teoría de Sistemas y Dinámica de Sistemas).

Con estas herramientas los alumnos y quienes quieran estudiar fenómenos urbanos en general tienen la posibilidad de pasar de una descripción verbal o imágenes a un modelo formal que permita evaluar la relación causal entre variables a partir de la comparación de diferentes escenarios.

Esta línea de investigación se enfoca en la producción de herramientas construidas desde lo más básico, para poder adaptarlas a las necesidades de investigación. Esto se ha observado en el curso, ya que los alumnos y otros docentes han propuesto modificaciones a los modelos, los cuales han resultado en versiones más completas y fieles a los fenómenos a estudiar.

También se han observado los beneficios de trabajar a partir de imágenes, a diferencia del trabajo en base a topologías (vectores). Los modelos a partir de Autómatas Celulares trabajan desde grillas o matrices que almacenan valores, lo cual es similar a una imagen raster (grilla de valores o pixeles que conforman una imagen). Esto hace posible trabajar a un nivel más básico, ingresando a los modelos imágenes producidas por los alumnos y facilita el trabajo en diferentes escenarios, ya que es posible modificar las imágenes en programas de edición tradicionales como Powerpoint o Photoshop y luego reingresar la imagen. Esto se diferencia del trabajo con archivos vectoriales (a partir de elementos con atributos, como AutoCad o $\operatorname{ArcG}(S)$, que requieren de mayor precisión en el ingreso y edición de información.

Por último, se debe recalcar la importancia de considerar los resultados de los modelos como una forma de entregar más antecendentes para la toma de decisiones, pero nunca como el reemplazo del sentido común y experiencia de usuario, considerando el modelamiento como una opinión más dentro del análisis general. Los modelos presentados no son herramientas de predicción, sino herramientas que permiten agregar y entender nuevas dimensiones de análisis.

\section{BIBLIOGRAFÍA}

Anas, A. (1982). Residential location markets and urban transportation: economic theory econometrics and policy analysis with discrete choice models. New York: Academic Press.

Axelrod, R. (1997). The Complexity of Cooperation. Agent Based models of Competition and Colaboration; Princeton University Press.

Barros, J. (2003). Simulating Urban Dynamics in Latin American Cities; Centre for Advanced Spatial Analysis proceedings. of the $7^{\text {th }}$ International Conference on GeoComputation (8-10 September 2003)

Batty, M. (1995). New ways of looking at cities. Nature, 377, 574

Ben Akiva, M \& Lerman, S. (1985). Discrete choice analysis: theory and application to travel demand. Cambridge: MIT Press. 
Benenson, I. (1998). Multiagent simulations of residential dynamics in the city; Comput., Environ. and Urban Systems, Vol. 22, № 1, pp. 25-42.

Borsdorf, A. (2003). Cómo modelar el desarrollo y la dinámica de la ciudad latinoamericana. EURE, 86, recuperado de www.scielo.cl/scielo.php? pid $=$ S0250-71612003008600002\&script $=$ sci_ arttext

Castoriadis, C. (1975) La institución imaginaria de la sociedad. Colección Fábulas, Ciencias Sociales. Barcelona: Tusquets Editores, $584 \mathrm{p}$.

De Mattos, C. (2001). Metropolización y Suburbanización. EURE, 80, Recuperado de http://www.scielo.cl/scielo.php?pid=S025071612001008000001 \&script=sci_arttext

Epstein, J.M. (2008). Why model? Journal of Artificial Societies and Social Simulation. 11, (4).

Filatova, T, Parker, D; \& van der Veen, A. (2009). Agent-Based Urban Land Markets: Agent's Pricing Behavior, Land Prices and Urban Land Use Change. Journal of Artificial Societies and Social Simulation 12(1)3 http://jasss.soc.surrey. ac.uk/12/1/3.html, 2009.

Florescano, E. $(1980,2005)$ De la memoria del poder a la historia como explicación. Pp. 91-128, Cap. en PEREYRA, Carlos et al. Historia, ¿̇para qué? Vigésimo primera edición. México: Siglo XXI Editores, S. A. de C. V., 249 p.

Gardner, Martin (1970). The fantastic combinations of John Conway's new solitaire game «life». Scientific American, 223, 120-123.

Harvey, D. (1998). La condición de la posmodernidad. Investigación sobre los orígenes del cambio cultural. Buenos Aires: Amorrortu Editores, $391 \mathrm{p}$.
Hotelling, H. (1929). «La estabilidad en la competencia»; En Stigler, Boulding, (1968). Ensayos sobre la teoría de los precios; Aguilar. Jacobs, J. (1961). The Death and Life of Great American Cities; Random House.

Keeney, R. \& Raiffa, H. (1976). Decisions with Multiple Objectives: Performances and Value Trade-Offs. Wiley, New York.

Krugman, P. (1991). Geography and Trade. Cambridge: The MIT Press.

Lindón, A. (2007). El constructivismo geográfico y las aproximaciones cualitativas. Pp. 5-11, Art. en Revista de Geografía Norte Grande, Núm. 37, junio del 2007. Santiago: Instituto de Geografía, Facultad de Historia, Geografía y Ciencia Política P. Universidad Católica de Chile / (2009). La construcción socioespacial de la ciudad: el sujeto cuerpo y el sujeto sentimiento. Pp. 6-20, Art. en Revista Latinoamericana de Estudios sobre Cuerpos, Emociones y Sociedad, Año 1, Núm. 1., diciembre 2009 - marzo 2010. Córdoba: Red Latinoamericana de Estudios Sociales sobre las Emociones y los Cuerpos / Programa de Acción Colectiva y Conflicto Social del CIECS (CONICET-UNC) / Grupo de Estudios sobre Sociología de las Emociones y los Cuerpos del IIGG-UBA.

Malczewski, J., \& Ogryczak, W. (1995). The multiple criteria location problem: 1. A generalized network model and the set of eficient solutions. Environment and Planning A, 27, 1931-1960.

Polhill, J. G; Sutherland L-A; Gotts, N. (2010). Using Qualitative Evidence to Enhance an AgentBased Modelling System for Studying Land Use Change. Journal of Artificial Societies and Social
Simulation 13 (2) $10 \mathrm{http}: / /$ jasss.soc.surrey. ac.uk/13/2/10.html 2010

Rigotti, A. M. (1996). Los usos de la ciencia. El expediente urbano en los primeros planes argentinos. IV Seminário de História da Cidade e do Urbanismo Vol. 4, № 1.

Rio de Janeiro, 1997. (http://hdl.handle. net/2133/2200)

Roy, B. \& Vanderpooten D. (1996). The European School of MCDA. Emergence, Basic Features, and Current Works. Journal of Multi-Criteria Decision Analysis 5: 22-37.

Ruiz-Tagle, J; Gurovich A. \& Cox T. (2009). Modelos de simulación urbana. Aproximación experimental a los fenómenos de la ciudad. Santiago: Facultad de Arquitectura y Urbanismo, Universidad de Chile, $129 p$

Saaty, T. L. (1980). The Analytic Hierarchy Process, Planning, Priority Setting, and Resource Allocation. New York, McGraw Hill.

Schelling, T. (1969). Models of segregation. The American Economic Review, 59(2), 488-493.

Waddell, P. (2009). Parcel-Level Microsimulation of Land Use and Transportation: The Walking Scale of Urban Sustainability. Paper para IATBR Workshop on Computational Algorithms and Procedures for Integrated Microsimulation Models, Jaipur, India.

Wegener, M. (2001). From Macro to Micro - How Much Micro is too Much? Transport Reviews 31 (2), 161-177.

Wilensky, U. (1999). NetLogo. http://ccl.northwestern. edu/netlogo/. Center for Connected Learning and Computer-Based Modeling, Northwestern University, Evanston, IL. 